Article

\title{
Comparing Pedophile Activity in Different P2P Systems
}

\author{
Raphaël Fournier ${ }^{1, *}$, Thibault Cholez ${ }^{2}$, Matthieu Latapy ${ }^{3,4}$, Isabelle Chrisment ${ }^{2}$, \\ Clémence Magnien ${ }^{3,4}$, Olivier Festor ${ }^{2}$ and Ivan Daniloff ${ }^{3,4}$
}

${ }^{1}$ L2TI/Institut Galilée, Université Paris-Nord, 99 avenue JB Clément, 93430 Villetaneuse, France

${ }^{2}$ LORIA/INRIA Nancy-Grand Est, 615 Rue du Jardin Botanique, 54600 Villers-lès-Nancy, France;

E-Mails: Thibault.Cholez@inria.fr (T.C.); Isabelle.Chrisment@loria.fr (I.C.);

Olivier.Festor@loria.fr (O.F.)

${ }^{3}$ Sorbonne Universités, UPMC Univ Paris 06, UMR 7606, LIP6, F-75005 Paris, France;

E-Mails: Matthieu.Latapy@lip6.fr (M.L.); clemence.magnien@lip6.fr (C.M.); ivan.daniloff@laposte.net (I.D.)

${ }^{4}$ CNRS, UMR 7606, LIP6, F-75005 Paris, France

* Author to whom correspondence should be addressed; E-Mail: raphael.fournier@univ-paris13.fr; Tel.: +33-149-402-824.

Received: 19 March 2014; in revised form: 15 May 2014 / Accepted: 16 May 2014 /

Published: 1 July 2014

\begin{abstract}
Peer-to-peer (P2P) systems are widely used to exchange content over the Internet. Knowledge of pedophile activity in such networks remains limited, despite having important social consequences. Moreover, though there are different P2P systems in use, previous academic works on this topic focused on one system at a time and their results are not directly comparable. We design a methodology for comparing KAD and eDonkey, two P2P systems among the most prominent ones and with different anonymity levels. We monitor two eDonkey servers and the $K A D$ network during several days and record hundreds of thousands of keyword-based queries. We detect pedophile-related queries with a previously validated tool and we propose, for the first time, a large-scale comparison of pedophile activity in two different P2P systems. We conclude that there are significantly fewer pedophile queries in $K A D$ than in eDonkey (approximately $0.09 \%$ vs. $0.25 \%$ ).
\end{abstract}

Keywords: P2P networks; eDonkey; pedophile activity 


\section{Introduction}

Pedophile activity is a crucial social issue and is often claimed to be prevalent in peer-to-peer (P2P) file-sharing systems [1,2]. However, current knowledge of pedophile activity in these networks remains limited.

Recently, research works have been conducted to improve this situation by quantifying pedophile activity in Gnutella and eDonkey, two of the main P2P systems currently deployed [3,4]. They conclude, respectively, that $1.6 \%$ and $0.25 \%$ of queries are of pedophile nature, but these numbers are not directly comparable as the authors use very different definitions and methods. Such comparisons are of high interest though, since differences in features of P2P systems, such as the level of anonymity they provide, may influence their appeal for pedophile users.

In this paper, we perform a comparison for the first time. We focus on the $K A D$ and $e$ Donkey $\mathrm{P} 2 \mathrm{P}$ systems, which are the names given to the two underlying P2P networks used by the popular eMule file-sharing application. They are both widely used, accounting together for almost $10 \%$ of the global Internet traffic in Europe in 2012 [5], but they differ significantly in their architecture: while eDonkey relies on a few servers, $K A D$ is fully distributed. This lack of centralization may lead users to assume that $K A D$ provides a much higher level of anonymity than eDonkey. Comparing the two systems sheds light on the influence of a distributed architecture on pedophile behavior and increases general knowledge on pedophile activity in $\mathrm{P} 2 \mathrm{P}$ systems.

The term pedophilia is popularly used to denote adult sexual engagement with children, both prepubescent and pubescent. The definition of pedophilia we use in this article thus encompasses both the medical definition of pedophilia (sexual interest in prepubescent children) and hebephilia (sexual interest in pubescent children not sexually mature).

We discuss related work in Section 2, to give an overview of the state-of-the-art on online pedophile activity detection and analysis. Section 3 presents a short introduction to P2P systems, before our description of our datasets and how we collected them (Section 4). We then present the details of our comparison of the amount of pedophile queries in KAD and eDonkey in Section 5. Section 6 focuses on an important feature of pedophile activity: ages entered in queries. Finally, in Section 7, we introduce a methodology to estimate the fraction of pedophile queries in $K A D$ from the one in eDonkey.

\section{Related Work}

Collecting P2P traces is an active topic for years, but it is mostly aimed at analyzing peer behavior to help with future P2P protocol design. In 2006, authors of [6] and [7] explored the social and technical issues related to online child pornography and opened the way to the research in the field. The first detailed quantitative study focusing on a P2P system was proposed in [3], using an active methodology (sending specific queries and analyzing the answers provided by the search engine). Since then, several approaches have been proposed to gage the extent of the phenomenon. Among them, [8] presented filename categorization tool, while $[9,10]$ proposed to label suspicious chat conversations. [11] especially analyzed aged-related queries.

A first large-scale study of P2P search-engine queries was presented in [12]. Their study focused on "onset", the first deliberate viewing of child pornography. They gathered the Top 300 queries submitted 
to the popular Isohunt tracker (part of the BitTorrent network) and published on the website Isohunt.com. Their study lasts for 3 months, a scale similar to ours, but they resort to manual classification of the queries. Their dataset is particular, as it only gives a relative popularity order for some queries, and may not provide any indication on the extent of child pornography in the network. Plus, with only 300 queries collected daily, they get very few pedophile queries (only 3), which leads to results with a limited statistical significance. However, their discussion is truly interesting, including comments on whether "regular pedophile users" are likely to submit several times the same query (to "build a collection"), while first-time users may not (they do not progress to downloading material once they have discovered the meaning of intriguing pedophile sequences such as pthc). This bias may lead query-based studies like ours to slightly overestimating the demand for child pornography, and would impact estimations on the number of pedophile users, but additional filtering based on the IP-address or the client ID could limit this issue.

In [4], the authors developed and assessed a dedicated tool for search engine query classification, and collected large-scale datasets on eDonkey (up to 28 weeks of uninterrupted experiment). We use here their tool and one of their datasets. Part of their work was later reused by another team to study another P2P network, BitTorrent [13]. The European Commission has set up a "Safer Internet" program [1], which funded some large research projects such as MAPAP [14] and iCOP [15].

In parallel, authors of [16,17] provided an extensive study (one-year long) on child pornography on Gnutella and eMule, partnering with law enforcement to develop software platforms and collect data on child pornography trafficking. They made a precious contribution to understand the "supply": how many users are involved in the distribution of files, what are their importance in the network, etc. In [16], they evaluated different strategies to best fight pedophile activity given the limited resources of law enforcement and proposed an efficient metric to target the most prominent peers.

While having a smaller scale, our study is the first to provide a methodology to gain new knowledge from the proper comparison of data collected from two P2P networks which architecture and monitoring capacity are totally different. Moreover, if the general user behavior in the $K A D$ network was detailed in [18], our article is the first to study whether its decentralized architecture is prone to favor criminal activity.

\section{P2P Systems}

P2P systems are computer networks in which every user may share content with others members. They have become popular because they gather large amount of digital contents (books, movies, music) which can be obtained for free. Copyrighted material is available (however not authorized) and pornography is widespread. Accessing a P2P network is generally easy: a user only needs to download and install on his computer a single application, which will handle the connection process to the network. Then, he can search for files with some keywords, and gets a list of corresponding available files. The application sends messages to the network to find providers of the selected files, and then users interconnect directly to exchange them. 
P2P networks are easy to access for both providers and consumers. Contents are obtained free of charge, and rather anonymously (no personal details are required). These features make such networks appealing for illegal activities such as pedophile material trafficking.

P2P networks account for approximately a fifth of the global bandwidth use on the Internet. Bittorrent is the most prominent $\mathrm{P} 2 \mathrm{P}$ network nowadays, preceding eDonkey and $K A D$ (the usage of which decline in Europe). For instance, an important $e$ Donkey server received on average 8.8 million queries per week between 2009 and 2012 [4].

\section{Experimental Setup and Datasets}

In order to compare pedophile activity in two different P2P systems, we first need appropriate datasets, the collection of which is a challenge in itself. In $K A D$ and $e$ Donkey, different kinds of measurements are possible, depending on the details of the network's architecture.

In eDonkey, servers index files and providers for these files, and users submit keyword-based queries to servers to seek files of interest to them [19]. By monitoring such a server, one may collect all those queries [20]. Here, we record all queries received by two of the largest eDonkey servers during a three-month period in 2010. The servers are located in different countries (France and Ukraine) and have different filtering policies: the French server indexes only non-copyrighted material, while the Ukrainian server openly indexes all submitted files. Monitoring two such different servers will allow us to compare them in order to know if server policy impacts our results.

To collect $K A D$ data, we use the HAMACK monitoring architecture [21], which makes it possible to record the queries related to a given keyword by inserting distributed probes close to the keyword ID onto the $K A D$ distributed hash table. We supervise 72 keywords, which we choose to span well the variety of search requests entered in the system, with a focus on pedophile activity: a set of 19 paedophile keywords (babyj, babyshivid, childlover, childporn, hussyfan, kidzilla, kingpass, mafiasex, pedo, pedofilia, pedofilo, pedoland, pedophile, pthc, ptsc, qqaazz, raygold, yamad, youngvideomodels), which are known to be directly and unambiguously related to pedophile activity in P2P networks; a set of 23 mixed keywords (1yo, 2yo, 3yo, 4yo, 5yo, 6yo, 7yo, 8yo, 9yo, 10yo, 11yo, 12yo, 13yo, 14yo, $15 y o, 16 y o$, boy, girl, mom, preteen, rape, sex, webcam) frequently used in pedophile queries but also in other contexts (for instance, Nyo stands for $N$ years old and is used by both pedophile users and parents seeking games for children of this age); and a set of 30 not paedophile keywords (avi, black, christina, christmas, day, doing, dvdrip, early, flowers, grosse, hot, house, housewives, live, love, madonna, man, new, nokia, pokemon, rar, remix, rock, saison, smallville, soundtrack, virtual, vista, windows, world) used as a test group and a priori rarely used in pedophile queries. The sets of keywords were established using the work on pedophile query detection presented in [4]. Notice that our set of keywords contains mainly common English words (love, early, flowers), but some are in other languages (saison, pedofilia), and some are also brand names (pokemon, nokia).

Because of the differences in architectures of the two networks and of the measurement methodologies, we obtained very different datasets, which are not directly comparable: in eDonkey, we observe all queries from a subset of users whereas in $K A D$ we only observe queries related to a given keyword, but from all users. In addition, based on various versions of $K A D$ clients, the measurement 
tool only records the queries containing a monitored keyword placed in first position or being the longest in the query. As a consequence, with a short keyword such as avi, a name extension for video files, we almost only record queries in which it is the unique keyword, because otherwise it most likely is neither the longest nor the first word in any query. In order to obtain comparable datasets, we therefore limit our study to a subset of our datasets: the queries composed of exactly one word among the 72 keywords we monitor.

As a result of this construction process, we obtain three datasets, which we call eDonkeyFR, eDonkeyUA and KAD. They contain 241,152, 166,154 and 250,000 queries respectively, all consisting of a unique keyword from our list of 72 monitored keywords, which ensures that they are comparable. The server corresponding to the $e D o n k e y F R$ dataset is located in France, while the one corresponding to $e D o n k e y U A$ is in Ukraine. Their large sizes make us confident in the reliability of our statistical results presented hereafter.

\section{Amount of Pedophile Queries in eDonkey versus KAD}

The most straightforward way to compare the pedophile activity in different systems certainly is to compare the fraction of pedophile queries in each system. Figure 1 presents the fraction of queries for each category of keywords. This plot clearly shows that there are very distinct search behaviors in the two networks, since values obtained for the paedophile and not paedophile categories significantly differ between $K A D$ and the two eDonkey datasets. More surprisingly, the fraction of pedophile queries is significantly lower in $K A D$ than in eDonkey which is in sharp contradiction with previous intuition, as $K A D$ is assumed to provide a higher level of anonymity. The plot also shows that values obtained for the two eDonkey servers are similar, which indicates that very different filtering policies have no significant influence on the amount of pedophile queries.

In order to gain a more detailed insight on this phenomenon, we study the frequencies of each keyword separately in the three datasets. As we want to explore possible correlations between the pedophile nature of a keyword and its frequency, we need a way to quantify the pedophile nature of a keyword. To do so, we use the 28-week dataset and the pedophile query detection tool from [4], which divides a dataset between paedophile and not paedophile queries (with a precision above $98 \%$ and a recall above 75\%). We denote by $Q$ the whole dataset of queries, and by $Q(k)$ the set of queries containing a given keyword $k$. For each keyword $k$, we obtain $Q(k)=N(k)+P(k)$, where $N(k)$ and $P(k)$ are the subset of queries containing keyword $k$ and tagged as not paedophile or paedophile, respectively. We then define the paedophile coefficient $\pi(k)$ of keyword $k$ as: $\pi(k)=\frac{|P(k)|}{|Q(k)|}$. If all the queries with keyword $k$ are pedophile queries, $\pi(k)=1$, and if none of them are, $\pi(k)=0$. All keywords in the not paedophile category have a paedophile coefficient below 0.006 . For keywords in the mixed category, the paedophile coefficient is above 0.01 and below 0.4. All paedophile keywords have a paedophile coefficient above 0.885 . Finally, we plot in Figure 2 the ratios $\frac{f_{e D o n k e y F R}(k)}{f_{k a d}(k)}$ and $\frac{f_{e D o n k e y U A}(k)}{f_{\text {kad }}(k)}$, where $f_{s}(k)$ denotes the frequency of queries composed of keyword $k$ in the dataset $s$, for each of our 72 keywords. We rank keywords on the horizontal axis in increasing order of paedophile coefficient. The horizontal line represents $y=1$, which enables a visual comparison of the values: if the point is below the line, then the keyword is more frequent in $K A D$, otherwise it is more frequent in the $e$ Donkey dataset. 
Figure 1. Fraction of queries of each kind in our three datasets.

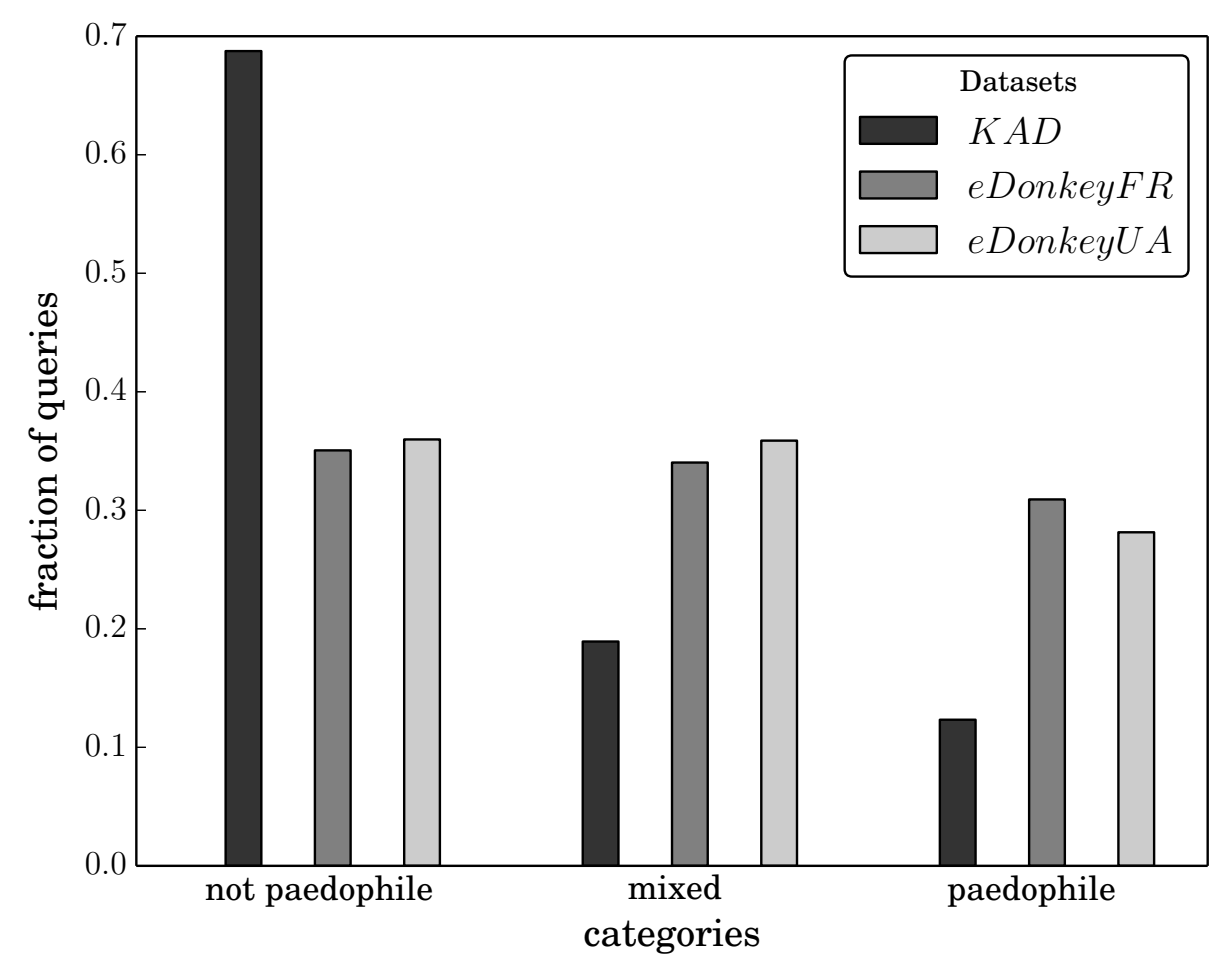

Figure 2. Ratio of keyword frequencies in eDonkey vs. KAD. Keywords are ranked in increasing order of paedophile coefficient. Points above the $y=1$ horizontal line indicates keywords more frequent in the corresponding eDonkey dataset; below the line keywords are more frequent in $K A D$.

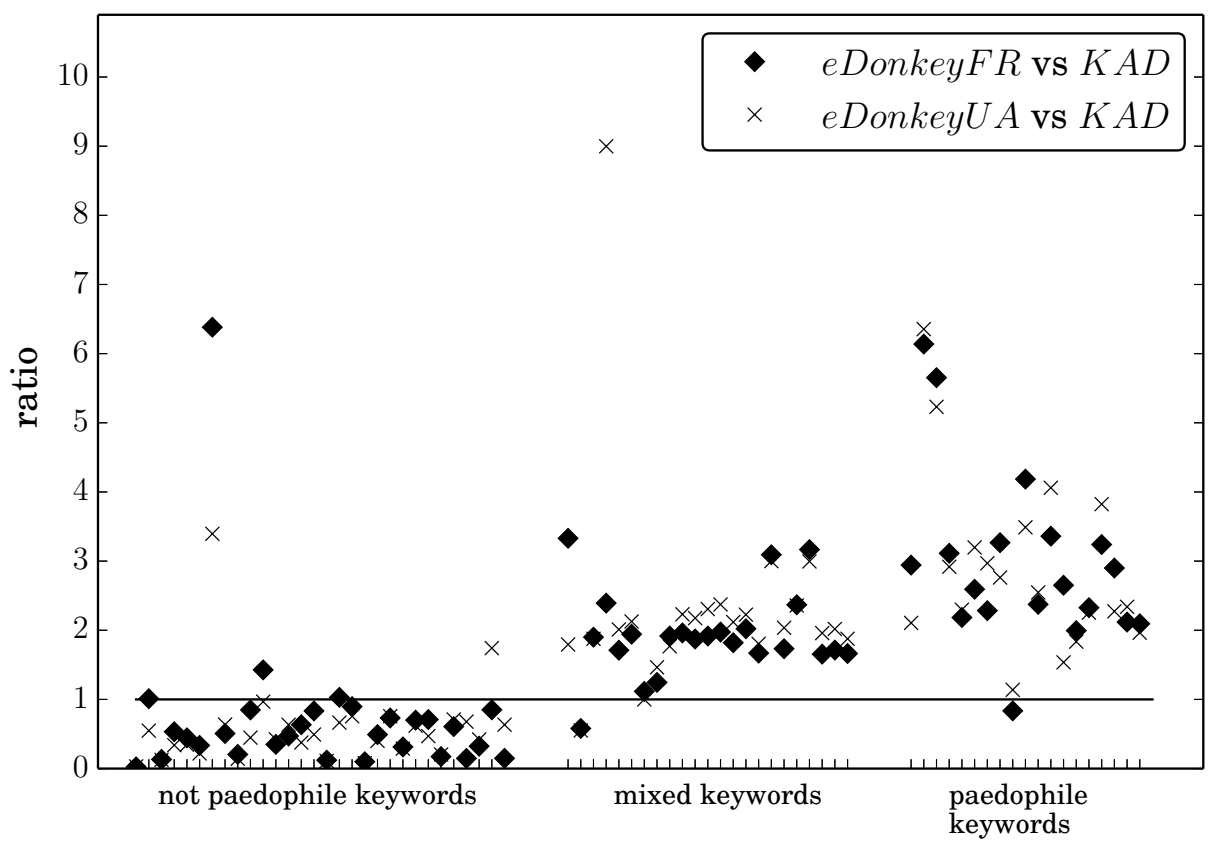

This plot gives a clear evidence for a correlation between the pedophile nature of a keyword and its higher presence in $e$ Donkey than in $K A D$. In addition, the frequencies in both $e$ Donkey datasets are very similar for the vast majority of keywords. 
We therefore conclude that anonymity is not the prevailing factor when pedophile users choose a network, since neither the decentralized architecture of $K A D$ nor the different filtering policies increase the frequency of pedophile queries. Instead, the frequency of pedophile queries is even higher in $e$ Donkey than in $K A D$. Finding an explanation for this unexpected phenomenon is still an open question. The higher technical skills required to use $K A D$ may be part of the explanation. Users may also search content on eDonkey while protecting their privacy with other tools, such as Virtual Private Networks or TOR [22]. The fact that in $K A D$ search requests are sent over UDP and cannot benefit from TOR anonymization could explain the difference in the network usage.

\section{Ages Indicators in Queries}

A way to gain more insight on observed pedophile activity is to study the distribution of age indicators in queries [11]. Notice that age indicators are sometimes used in other contexts than pedophile activity, especially when parents seek content suitable for children of a certain age. However, one can observe on Figure 2 that ages indicators have similar behavior to those obtained for the pedophile group, and are therefore closely related to the topic.

We plot the distribution of age indicators on Figure 3: for each integer $n$ lower than 17, we plot the number of queries of the form nyo in each dataset (yo stands for years old). The three plots have similar shape, with mostly increasing values from 1 to 10, a little drop at 11, a peak at 12 and a fall from 13 to 16. These values for $K A D$ are below the values for the $e$ Donkey servers, which is due to the fact that this dataset is a bit smaller than others and that pedophile queries are rarer in it. The key point here is that the distributions are very similar in all three datasets. This indicates that, although the amount of pedophile activity varies between systems, its nature is similar, at least regarding ages.

Figure 3. Distribution of age indicators in our three datasets.

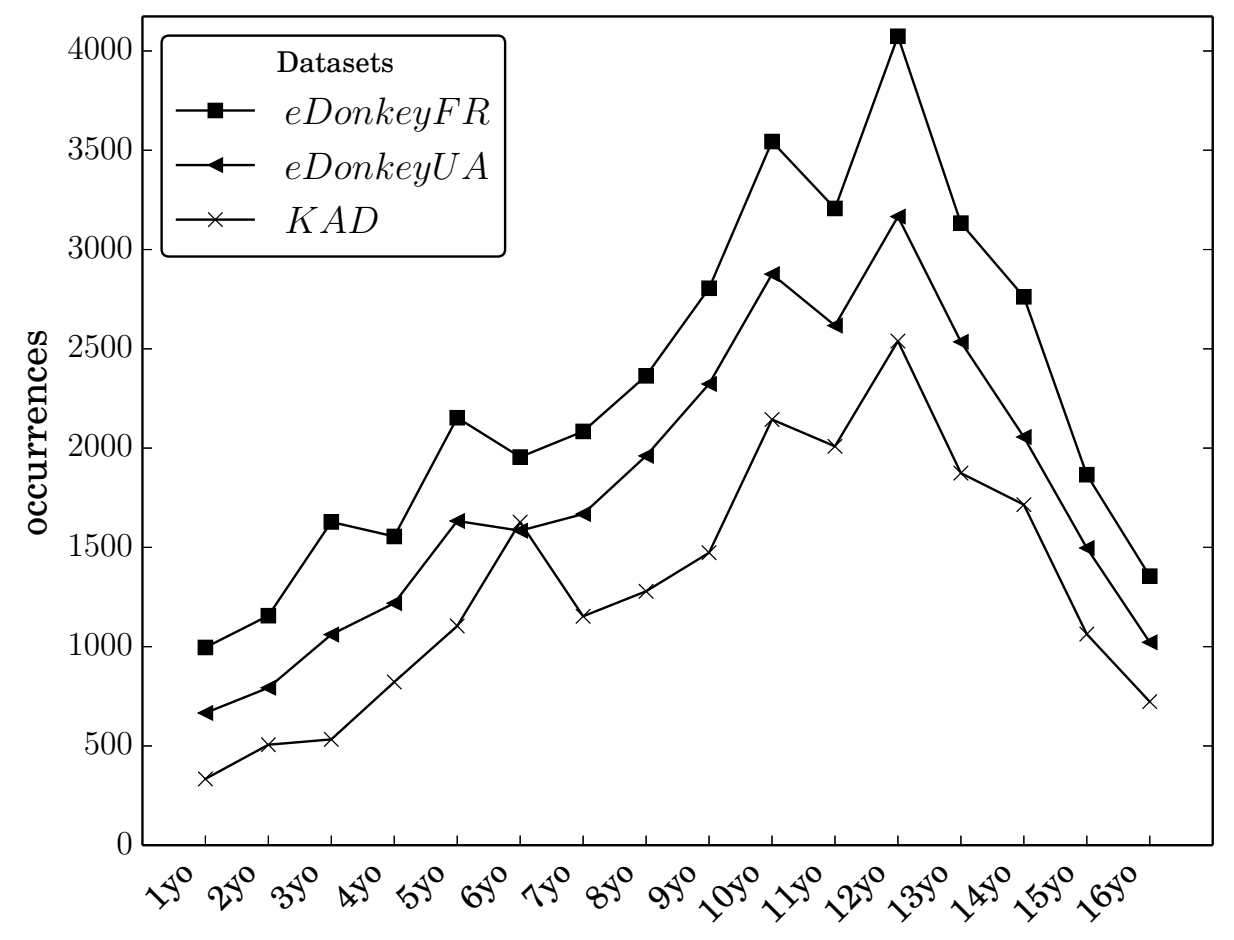




\section{Quantifying Pedophile Activity in $K A D$}

In [4], the authors establish a method to quantify the fraction of pedophile queries in eDonkey. It relies on a tool able to accurately tag queries as pedophile or not, and on an estimate of the error rate of this tool. Such an approach cannot directly be applied to $K A D$ though, as only a small (and biased) fraction of all queries may be observed in this system. We however show in this section how to derive the fraction of pedophile queries in $K A D$ from the one in $e$ Donkey.

In a given system, $e$ Donkey or $K A D$ here, we consider different sets of queries and we denote by $Q$ the set of all queries, $P$ the subset of pedophile queries in $Q, \bar{Q}$ the subset of queries composed of one word among the 72 monitored keywords, $\bar{P}$ the subset of pedophile queries with one word, i.e. consisting of one of the 19 monitored pedophile keywords (and so: $\bar{P}=\bar{Q} \cap P$ ). Figure 4 illustrates our notations.

In both our eDonkey measurements, $|P|$ and $|Q|$ may be directly estimated, as shown in [4], and one can then obtain the fraction $\frac{|P|}{|Q|}$ of pedophile queries in the dataset. We give the results for our two measurements in Table 1 . On the contrary, in $K A D$, one may only estimate $|\bar{P}|$ and $|\bar{Q}|$.

Figure 4. The different sets of queries defined for each dataset.

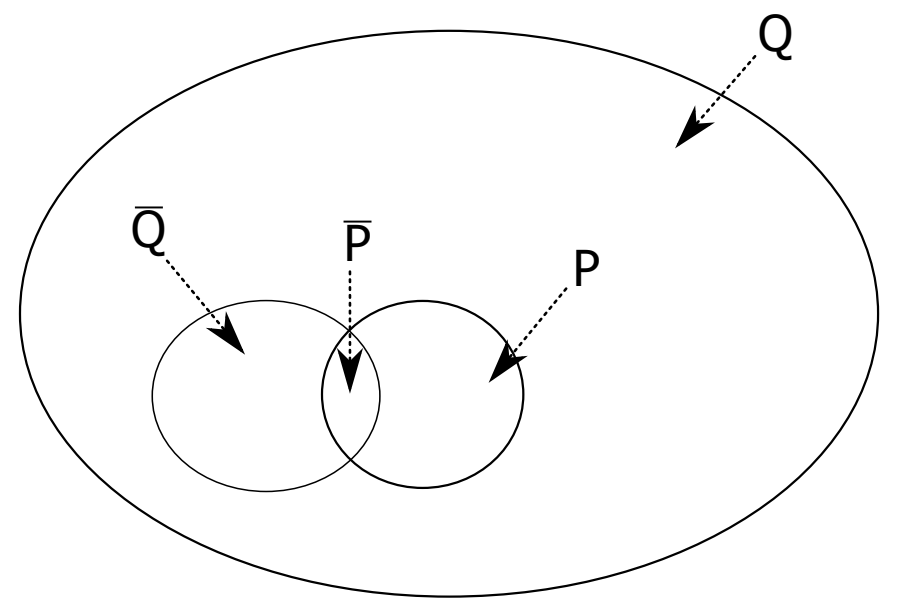

Table 1. Results for our three datasets.

\begin{tabular}{cccccc}
\hline Dataset & $\frac{|P|}{|Q|}$ & $|\bar{P}|$ & $|\bar{Q}|$ & $\alpha$ & $\beta$ \\
\hline edonkeyFR & $2.554 \cdot 10^{-3}$ & 74,557 & 241,152 & $1.431 \cdot 10^{-3}$ & 0.2502 \\
edonkeyUA & $2.668 \cdot 10^{-3}$ & 46,763 & 166,154 & $1.538 \cdot 10^{-3}$ & 0.2251 \\
$K A D$ & $\mathrm{n} / \mathrm{a}$ & 30,821 & 250,000 & $\mathrm{n} / \mathrm{a}$ & $\mathrm{n} / \mathrm{a}$ \\
\hline
\end{tabular}

However, we define $\alpha=\frac{|\bar{Q}|-|\bar{P}|}{|Q|-|P|}$ and $\beta=\frac{|\bar{P}|}{|P|}$, which capture the probability for a non pedophile query, respectively pedophile, to make a query of one word among one of our monitored keywords. Given the definition of $\alpha$ and $\beta$, there is no a priori reason to assume that they have significantly different values between $e$ Donkey and $K A D$. From the definitions of $\alpha$ and $\beta$, we have: 


$$
\begin{gathered}
\alpha=\frac{|\bar{Q}|-|\bar{P}|}{|Q|-|P|} \quad \Longrightarrow \quad Q \mid=\frac{\alpha|P|+|\bar{Q}|-|\bar{P}|}{\alpha} \\
\beta=\frac{|\bar{P}|}{|P|} \quad \Longrightarrow \quad P \mid=\frac{|\bar{P}|}{\beta}
\end{gathered}
$$

Then, the following expression holds:

$$
\begin{aligned}
\frac{|P|}{|Q|} & =\frac{|\bar{P}|}{\beta} \times \frac{\alpha}{\alpha|P|+|\bar{Q}|-|\bar{P}|} \\
& =\frac{\alpha|\bar{P}|}{\beta|\bar{Q}|+(\alpha-\beta)|\bar{P}|}
\end{aligned}
$$

We now use expression (1) to infer the fraction of paedophile queries that were submitted in the $K A D$ P2P network during our experiment. Using the values from Table 1 and the average values of $\alpha$ and $\beta$ between our eDonkey datasets, we obtain:

$$
\frac{|P|}{|Q|} \approx 0.087 \% \pm 0.008
$$

This value is of similar magnitude to the one of eDonkey (approx. 0.25\%) but close to three times lower.

This estimation of $\frac{|P|}{|Q|}$ relies on the value of $\alpha$. One may wonder whether the choice of keywords from which we built $\bar{Q} \backslash \bar{P}$ has a significant impact on the estimated value of $\frac{|P|}{|Q|}$ in $K A D$. We check this as follows: we randomly select 1,000 subsets of 26 keywords out of the 53 keywords which compose the queries in $\bar{Q} \backslash \bar{P}$. We then compute, for each subset, the number of queries consisting of exactly one of those keywords and the resulting value of alpha. For $e D o n k e y F R$, we obtain an average value of $\bar{\alpha}=0.000889$ (minimum: 0.000256, maximum: 0.00153, and 90\% of the values in [0.000463;0.00133]). For $e$ DonkeyUA, we obtain an average value of $\bar{\alpha}=0.00105$ (minimum: 0.000352, maximum: 0.00172, and $90 \%$ of the values in $[0.00062 ; 0.00148]$ ). This means that we would obtain very similar results with 26 keywords only and so we may be confident in our estimate obtained with 53 keywords.

\section{Conclusions}

We performed a comparative study of two large-scale peer-to-peer networks, KAD and eDonkey, with regards to the queries related to child pornography. We designed a methodology to collect and process datasets allowing to compare them in a relevant manner. We obtained the counter-intuitive result that pedophile keywords are significantly more present in eDonkey than in $K A D$, despite the higher anonymity level it provides. On the contrary, our study of age indicators in queries showed that the nature of pedophile queries is similar in these systems. We finally established the first estimate of the fraction of pedophile queries in $K A D$. We obtained a value close to $0.09 \%$, which is of the same magnitude but significantly lower than in eDonkey $(0.25 \%)$.

Our approach here is similar to the one used in [4]: we focus on search queries, which help to grasp the demand for pedophile material. It differs from [16,17] which focused on the files. In P2P networks such as $e$ Donkey and $K A D$, a single file may have several names, most of which describe its content. However, filenames are prone to pollution and often exhibit keywords unrelated to the real content of 
the file, for instance a pedophile file may have a non-pedophile name [23,24]. Thus, estimations relying on specific filenames are likely to underestimate the true extent of child pornography distribution, while estimations relying on file-based honeypots are likely to overestimate the demand due to false-positive download requests. Query-based estimations using search requests do not suffer from such a bias, but, as mentioned earlier, may be impacted by repetitive queries from regular pedophile users. Nevertheless, both the considered P2P networks (KAD and eDonkey) should be equally affected, thus making their comparison valid to this regard.

Our contributions open various directions for future work. In particular, our methodology may be applied to compare other systems, and our datasets may be used to perform either deeper analyses on pedophile activity or on general search engine behaviors.

\section{Acknowledgments}

This work is supported in part by the MAPAP SIP-2006-PP-221003 and ANR MAPE projects.

\section{Author Contributions}

RF participated in the study design, carried out analyses and interpreted the data, before writing the first manuscript in consultation with the co-authors. TC, IC and OF participated in the study design, data collection and experiment analysis. ML and CM participated in the study design and data analysis. ID was of significant help to collect data.

\section{Conflicts of Interest}

The authors declare no conflict of interest.

\section{References}

1. European Commission. "Safer Internet Programme: Empowering and Protecting Children Online.” 2010. Available online: http://ec.europa.eu/information_society/activities/sip/ index_en.htm (accessed on 4 June 2014).

2. Declan McCullagh. "RIAA: Child porn rife on P2P networks." CNet, 9 September 2003. Available online: http://news.cnet.com/RIAA-Child-porn-rife-on-P2P-networks/ 2100-1028_3-5073817.html (accessed on 4 June 2014).

3. Daniel Hughes, James Walkerdine, Geoff Coulson, and Stephen Gibson. "Peer-to-Peer: Is Deviant Behavior the Norm on P2P File-Sharing Networks?" IEEE Distributed Systems Online 7 (2006): 1-11.

4. Matthieu Latapy, Clémence Magnien, and Raphaël Fournier. "Quantifying paedophile activity in a large P2P system.” Information Processing \& Management 49 (2013): 248-63.

5. Sandvine Network. "Global Internet Phenomena Report: Spring 2011." 2012. Available online: http://fr.scribd.com/doc/94722096/Sandvine-Global-Internet-Phenomena-Report-1H-2012 (accessed on 4 June 2014). 
6. Munish Chopra, Miguel Vargas Martin, Luis Rueda, and Patrick C. K. Hung. "Toward New Paradigms to Combating Internet Child Pornography." Paper Presented at Canadian Conference on Electrical and Computer Engineering (CCECE), Ottawa, ON, Canada. IEEE, 7-10 May 2006, pp. 1012-15.

7. Asaf Shupo, Miguel Vargas Martin, Luis Rueda, Anasuya Bulkan, Yongming Chen, and Patrick C.K. Hung. "Toward efficient detection of child pornography in the network infrastructure." IADIS International Journal on Computer Science and Information Systems 1 (2006): 15-31.

8. Alexander Panchenko, Richard Beaufort, Hubert Naets, and Cedrick Fairon. "Towards Detection of Child Sexual Abuse Media: Categorization of the Associated Filenames." In Advances in Information Retrieval. Edited by Pavel Serdyukov, Pavel Braslavski, Sergei O. Kuznetsov, Jaap Kamps, Stefan Rüger, Eugene Agichtein, Ilya Segalovich and Emine Yilmaz. Lecture Notes in Computer Science. Springer: Berlin Heidelberg, Germany, 2013, vol. 7814, pp. 776-79.

9. April Kontostathis, Andy Garron, Kelly Reynolds, Will West, and Lynne Edwards. "Identifying Predators Using ChatCoder 2.0.” Paper Presented at CLEF Conference 2012 Evaluation Labs and Workshop, Rome, Italy, 17-20 September, 2012. Edited by Pamela Forner, Jussi Karlgren and Christa Womser-Hacker.

10. Nick Pendar. "Toward Spotting the Pedophile Telling Victim from Predator in Text Chats." Paper Presented at International Conference on Semantic Computing, Irvine, CA, USA, 17-19 September 2007, pp. 235-41.

11. Chad M.S. Steel. "Child pornography in peer-to-peer networks." Child Abuse \& Neglect 33 (2009): 560-68.

12. Jeremy Prichard, Paul A Watters, and Caroline Spiranovic. "Internet subcultures and pathways to the use of child pornography." Computer Law \& Security Review 27 (2011): 585-600.

13. Moshe Rutgaizer, Yuval Shavitt, Omer Vertman, and Noa Zilberman. "Detecting Pedophile Activity in BitTorrent Networks." In Passive and Active Measurement (PAM). Edited by Nina Taft and Fabio Ricciato. Lecture Notes in Computer Science. Springer: Berlin Heidelberg, Germany, 2012, vol. 7192, pp. 106-15.

14. MAPAP project. 2010. Available online: http://antipaedo.lip6.fr (accessed on 4 June 2014).

15. iCOP. 2014. Available online: http://scc-sentinel.lancs.ac.uk/icop/ (accessed on 4 June 2014).

16. Janis Wolak, Marc Liberatore, and Brian Neil Levine. "Measuring a year of child pornography trafficking by US computers on a peer-to-peer network." Child Abuse \& Neglect 38 (2013): 347-56.

17. Ryan Hurley, Swagatika Prusty, Hamed Soroush, Robert J. Walls, Jeannie Albrecht, Emmanuel Cecchet, Brian Neil Levine, Marc Liberatore, Brian Lynn, and Janis Wolak. "Measurement and analysis of child pornography trafficking on P2P networks." Paper Presented at International World Wide Web Conference (WWW), Rio de Janeiro, Brazil, 13-17 May 2013. Edited by Daniel Schwabe, Virgilio A. F. Almeida, Hartmut Glaser, Ricardo A. Baeza-Yates and Sue B. Moon. pp. 631-42.

18. Thomas Locher, David Mysicka, Stefan Schmid, and Roger Wattenhofer. "A Peer Activity Study in eDonkey and Kad.” Paper Presented at International Workshop on Dynamic Networks: Algorithms and Security (DYNAS), Wroclaw, Poland, 5 September 2009. 
19. Oliver Heckmann, Axel Bock, Andreas Mauthe, and Ralf Steinmetz. "The eDonkey File-Sharing Network." Paper presented at Jahrestagung der Gesellschaft für Informatik, Ulm, Germany, 20-24 September 2004. Edited by Peter Dadam and Manfred Reichert. Lecture Notes in Informatics; vol. 51, pp. 224-28.

20. Frédéric Aidouni, Matthieu Latapy, and Clémence Magnien. "Ten weeks in the life of an eDonkey server." Paper Presented at International Workshop on Hot Topics in P2P Systems, in conjunction with 23rd IEEE International Symposium on Parallel and Distributed Processing, IPDPS 2009, Rome, Italy, 23-29 May 2009.

21. Thibault Cholez, Isabelle Chrisment, and Olivier Festor. "Monitoring and Controlling Content Access in KAD." Paper presented at International Conference on Communications - ICC 2010 IEEE, Cape Town, South Africa, 23-27 May 2010.

22. The Tor Project, Inc. TOR project, 2012. Available online: https://www.torproject.org/ (accessed on 4 June 2014).

23. Guillaume Montassier, Thibault Cholez, Guillaume Doyen, Rida Khatoun, Isabelle Chrisment, and Olivier Festor. "Content Pollution Quantification in Large P2P networks: A Measurement Study on KAD.” Paper presented at 11th IEEE International Conference on Peer-to-Peer Computing (IEEE P2P'11); IEEE Communications Society, Kyoto, Japan, 31 August-2 September 2011; pp. 30-33. Projects: ANR MAPE and GIS 3SGS ACDAP2P.

24. Maggie Brennan, and Sean Hammond. "Complete critical literature review." iCOP: Identifying and Catching Originators in P2P Networks. Technical report, 2011. Available online: http://scc-sentinel.lancs.ac.uk/icop/sites/scc-sentinel.lancs.ac.uk.icop/files/ D4.1LiteratureReview.pdf (accessed on 4 June 2014).

(C) 2014 by the authors; licensee MDPI, Basel, Switzerland. This article is an open access article distributed under the terms and conditions of the Creative Commons Attribution license (http://creativecommons.org/licenses/by/3.0/). 